\title{
Experimental study of wall boundary conditions for large-eddy simulation
}

\author{
By IVAN MARUSIC ${ }^{1}$, GARY J. KUNKEL ${ }^{1}$ \\ AND FERNANDO PORTÉ-AGEL ${ }^{2}$ \\ ${ }^{1}$ Department of Aerospace Engineering and Mechanics, University of Minnesota, Minneapolis, \\ MN 55455, USA \\ ${ }^{2}$ St Anthony Falls Laboratory, University of Minnesota, Minneapolis, MN 55414, USA
}

(Received 7 May 2001 and in revised form 14 May 2001)

An experimental investigation was conducted to study the wall boundary condition for large-eddy simulation (LES) of a turbulent boundary layer at $R_{\theta}=3500$. Most boundary condition formulations for LES require the specification of the instantaneous filtered wall shear stress field based upon the filtered velocity field at the closest grid point above the wall. Three conventional boundary conditions are tested using simultaneously obtained filtered wall shear stress and streamwise and wallnormal velocities, at locations nominally within the log region of the flow. This was done using arrays of hot-film sensors and $\times$-wire probes. The results indicate that models based on streamwise velocity perform better than those using the wall-normal velocity, but overall significant discrepancies were found for all three models. A new model is proposed which gives better agreement with the shear stress measured at the wall. The new model is also based on the streamwise velocity but is formulated so as to be consistent with 'outer-flow' scaling similarity of the streamwise velocity spectra. It is therefore expected to be more generally applicable over a larger range of Reynolds numbers at any first-grid position within the log region of the boundary layer.

\section{Introduction}

As LES is being developed several issues continue to challenge the application of this form of simulation to high Reynolds number turbulent boundary layers. Some of these challenges and the role of experimentation in overcoming them were discussed in a recent workshop (Adrian et al. 2000). One of the outstanding issues identified was the specification of the wall boundary condition. In an LES of a high Reynolds number boundary layer the grid size, and thus the smallest resolved scale, is relatively large when compared to the smallest scales of the flow. The first grid point in the simulation is usually in the logarithmic layer well above the viscous sublayer. Thus, the sublayer is not resolved and the boundary condition must account for all of the effects of this small-scale near-wall turbulence. Because of the large amount of turbulent interaction in the region between the first grid point and the wall, the typical no-slip boundary condition is often replaced with a condition on the wall shear stress. Historically, this condition is based on the correlation between the local fluctuating shear stress at the wall and the velocity at the nearest grid point above the wall.

This study considers the $(1,3)$ component of three wall shear stress models used in LES, where 1,2,3 represent the streamwise $(x)$, spanwise $(y)$, and wall-normal $(z)$ 
directions respectively. The three models considered were developed by Schumann (1975), Grötzbach (1987) and Piomelli et al. (1989) and following the convention of Piomelli et al. (1989) will be referred to as the 'SG', 'shifted SG', and 'ejection' models. Piomelli et al. (1989) tested these models by comparing mean velocity profiles, second-order moments and mean wall shear stress from the LES, with corresponding results from direct numerical simulation (DNS). The flow considered was a turbulent channel flow at $\operatorname{Re}_{\tau}=U_{\tau} \delta / v=640$, where $\delta$ is channel half-width, $U_{\tau}$ is wall shear velocity and $v$ is kinematic viscosity. From their analysis the shifted SG and ejection models were found to yield the best results. It should be emphasized that these three models assume that a standard logarithmic law exists and have been used extensively in both attached and separated boundary layer calculations, even though the law of the wall is not valid for the latter. In this paper we restrict our attention to attached boundary layers. Recent attempts to develop wall shear stress models for complex flows where the law of the wall does not hold, including separated flows, are not considered here. A recent review of such models is given by Cabot \& Moin (2000).

In this experimental study, a turbulent boundary layer is considered at $R e_{\tau}=1350$. The modelled wall shear stress, obtained from the filtered velocity measurements from three $\times$-wires, is compared to the measured filtered (resolved) wall shear stress obtained from nine shear stress sensors. Correlation coefficients and wall shear stress energy spectra are used to determine which model more closely describes the filtered shear stress on the wall. Based on these comparisons a new model is proposed.

\section{Wall shear stress models}

A typical wall shear stress model used in LES is the Schumann-Grötzbach (SG) model,

$$
\tau_{m}(x, y, z, t)=\left[\frac{\left\langle\tau_{w}\right\rangle}{\langle\bar{u}(x, y, z, t)\rangle}\right] \bar{u}(x, y, z, t),
$$

where $\tau_{m}$ is the instantaneous modelled wall shear stress, $\left\langle\tau_{w}\right\rangle$ is the mean wall shear stress, $u$ is the instantaneous streamwise velocity component, $\left(^{-}\right)$denotes the spatial filtering operation, and \langle\rangle indicates a long time average. In this paper all wall shear stresses are kinematic stresses and the sign convention is as used in LES, i.e. $-\left\langle\tau_{w}\right\rangle$ has a positive value. This model forces the mean of the instantaneous filtered velocity to equal the determined mean of the actual wall shear stress. Schumann (1975) first used this condition in a simulation of a turbulent channel flow. The mean velocity, $\langle\bar{u}(x, y, z)\rangle$, was determined using the logarithmic law of the wall while the mean wall shear stress was calculated from the driving pressure gradient. Later, Grötzbach (1987) used the same model except that the mean velocity was calculated over the plane at position $z$ parallel to the wall and the mean wall shear stress was determined from the logarithmic law of the wall.

A modified version of the SG model is the shifted SG model,

$$
\tau_{m}(x, y, z, t)=\left[\frac{\left\langle\tau_{w}\right\rangle}{\langle\bar{u}(x, y, z, t)\rangle}\right] \bar{u}\left(x+\Delta_{s}, y, z, t\right),
$$

where $\Delta_{s}$ is a streamwise displacement. Here the instantaneous filtered velocity signature is shifted in the streamwise direction to better correlate the instantaneous shear stress at the wall with the instantaneous velocity above the wall. This agrees well with the theory of inclined coherent structures along the wall. The displacement is found from experiments and DNS. Piomelli et al. (1989) found the shifted SG model to be an 


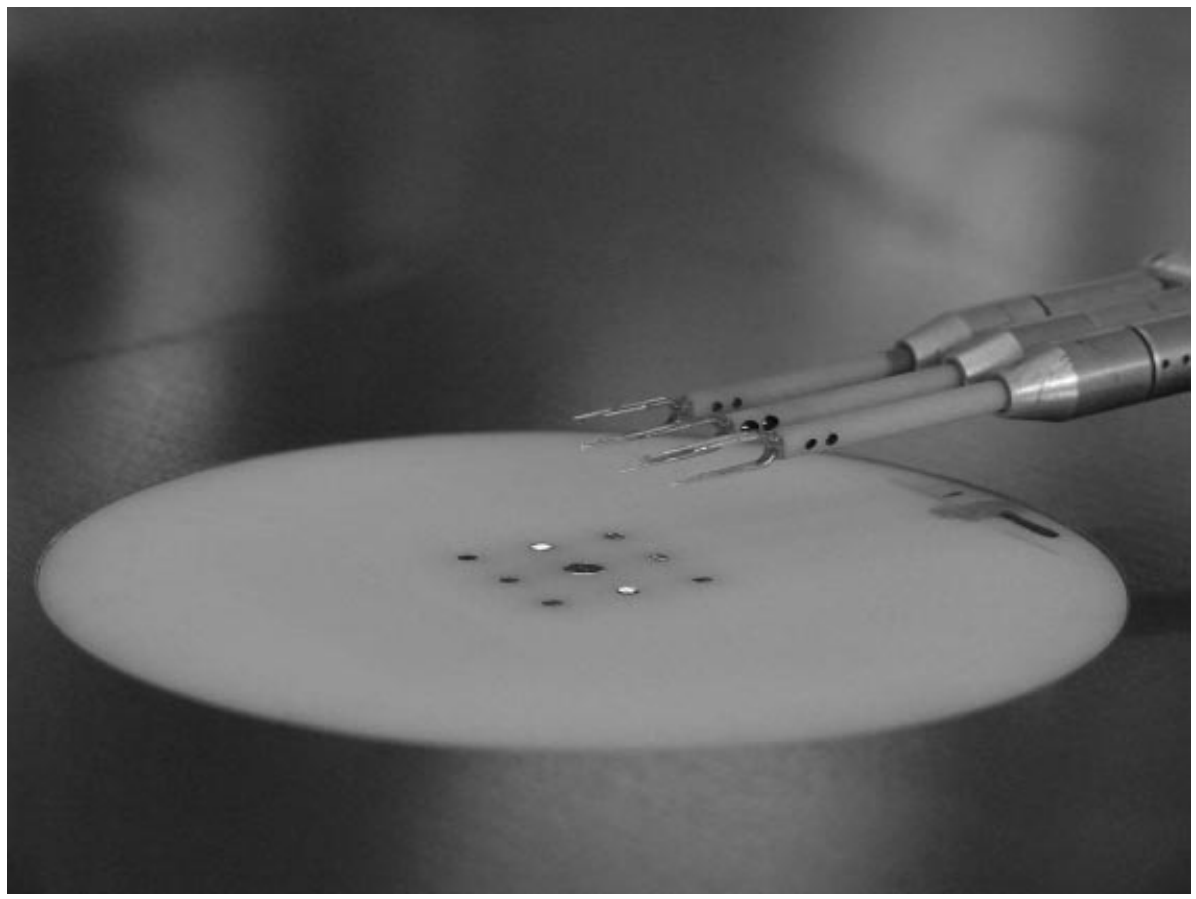

FigURE 1. View of experimental setup.

improvement over the standard SG model. They suggest using $\Delta_{s}=(1-|z|) \cot \left(8^{\circ}\right)$ for $30<z^{+}<50-60$, and that for larger distances from the wall the correlation between the shear stress at the wall and the velocity reaches a maximum along a plane inclined at $13^{\circ}$ to the wall.

Unlike the previous models, the ejection model uses the wall-normal component of the fluctuating velocity such that

$$
\tau_{m}(x, y, z, t)=\left\langle\tau_{w}\right\rangle+C U_{\tau} \bar{w}\left(x+\Delta_{s}, y, z, t\right),
$$

where $U_{\tau}$, the wall shear velocity, is equal to $\left(-\left\langle\tau_{w}\right\rangle / \rho\right)^{1 / 2}$ ( $\rho$ is the density of the fluid), $\bar{w}$ is the filtered wall-normal velocity component, and $C$ is a dimensionless constant of order 1 . The ejection model was suggested by Piomelli et al. (1989) to better describe the sweeping and ejection of vortex structures in the turbulent boundary layer. It was hypothesized that wall-normal velocity would better characterize the shear stress events at the wall.

\section{Experimental setup}

The experiment was conducted in a boundary layer wind tunnel with nominally zero streamwise pressure gradient where the working section was $1.2 \mathrm{~m}$ wide, $4.7 \mathrm{~m}$ long, and nominally $0.3 \mathrm{~m}$ high. Measurements were made at a location $3.2 \mathrm{~m}$ downstream of a trip-wire where the boundary layer thickness $\delta$ was $64 \mathrm{~mm}$. The Reynolds number based on momentum thickness $R_{\theta}=U_{1} \theta / v=3500$ where $\theta$ is momentum thickness and $U_{1}=8.9 \mathrm{~m} \mathrm{~s}^{-1}$ is the free stream velocity. This corresponds to a Kármán number $R e_{\tau}=U_{\tau} \delta / v=1350$. The temperature in the test section was monitored throughout the calibration and data acquisition and did not fluctuate by more than $\pm 0.5^{\circ} \mathrm{C}$.

Instantaneous wall shear stress measurements were obtained using nine TSI flush 
mounted hot-film sensors. Figure 1 shows a view of the experimental setup. Eight of the sensors were Model 1268, $1.5 \mathrm{~mm}$ diameter, and one was Model 1237, $3.2 \mathrm{~mm}$ diameter. The sensing films on all probes were $0.15 \times 1.5 \mathrm{~mm}$ (approximately $3 \times 30$ viscous wall units). They were operated with an AA Labs 10 channel anemometer at an overheat ratio of 1.5. All sensors were essentially a quartz cylinder with a sensing film mounted on one end of the cylinder. The nine sensors were mounted flush with the top of a $76 \mathrm{~mm}$ diameter Delrin plug in a $3 \times 3$ array with a distance of $6.02 \mathrm{~mm}$ between adjacent sensor centres. The plug was inserted through the bottom boundary wall. The plug was machined smooth and to the same height as the tunnel floor so that the sensors, the plug and the tunnel floor were all flush.

Instantaneous streamwise and wall-normal velocity measurements were obtained using three Auspex A55P51 $\times$-wire probes held in Dantec 55H24 probe supports. The wires were made and mounted in the laboratory and were $5 \mu \mathrm{m}$ copper coated tungsten wires with a $1 \mathrm{~mm}$ sensing length. They were operated with TSI IFA-100 anemometers at an overheat ratio of 1.7. As seen in figure 1 the wires were mounted in an array in the spanwise direction above the last downstream set of shear sensors. A stepper motor traverse was used to place the array of wires at the desired wallnormal positions. For the results presented here the wall normal wire positions were $z^{+}=z U_{\tau} / v=98,123,155,196,247$, and 311. All sensor signals were conditioned with a Tektronix VX4780 signal conditioner and digitized with a Tektronix VX4244 16-bit resolution digitizer. During data collection the nine wall shear stress sensors and six velocity sensor $(3 \times$-wires $)$ voltage signatures were sampled simultaneously at a rate of $10 \mathrm{kHz}$ for a record length totalling $105 \mathrm{~s}$. Velocity profiles across the entire boundary layer were obtained to ensure a standard boundary layer.

\subsection{Sensor calibration and correction}

Calibration of all sensors was performed at the beginning of each experimental run. The three $\times$-wires were calibrated in a separate calibration facility against a Pitot-static probe, at nine angles for each of seven velocities. A polar look-up table calibration method was then used to determine the two instantaneous velocity components from the two instantaneous voltages signatures.

The nine wall shear stress sensors were calibrated in-situ against a Preston tube connected to the same pressure transducer as above. A fourth-order polynomial curve fit was used and effects of higher-order moment corrections (as suggested by Breuer 1995) were checked and found to be negligible. The Delrin plug was allowed to reach thermal equilibrium between individual calibration points and before measurements were taken.

It is well known that the use of statically calibrated hot-film probes in air mounted on or in a wall substrate is plagued with complications resulting in inaccurate measurement of the fluctuating component of the wall shear stress. Experimental studies by Chew et al. (1998), Khoo et al. (1998), and Alfredsson et al. (1988) have shown that using film probes in this manner results in an attenuation of the amplitude of the fluctuating wall shear stress. They all suggest that a more accurate determination of instantaneous wall shear stress is obtained using a slightly elevated hot wire. However, because of the geometry of the $3 \times 3$ array using elevated hot wires is not a viable alternative.

A correction scheme was developed for the hot-film shear stress measurements based on obtaining the 'correct' power spectral density, for want of a better method. The reference spectrum was measured using an elevated hot wire within the viscous sublayer $\left(z_{+}=3.6\right)$. The amplitudes of each frequency component of the Fourier series 


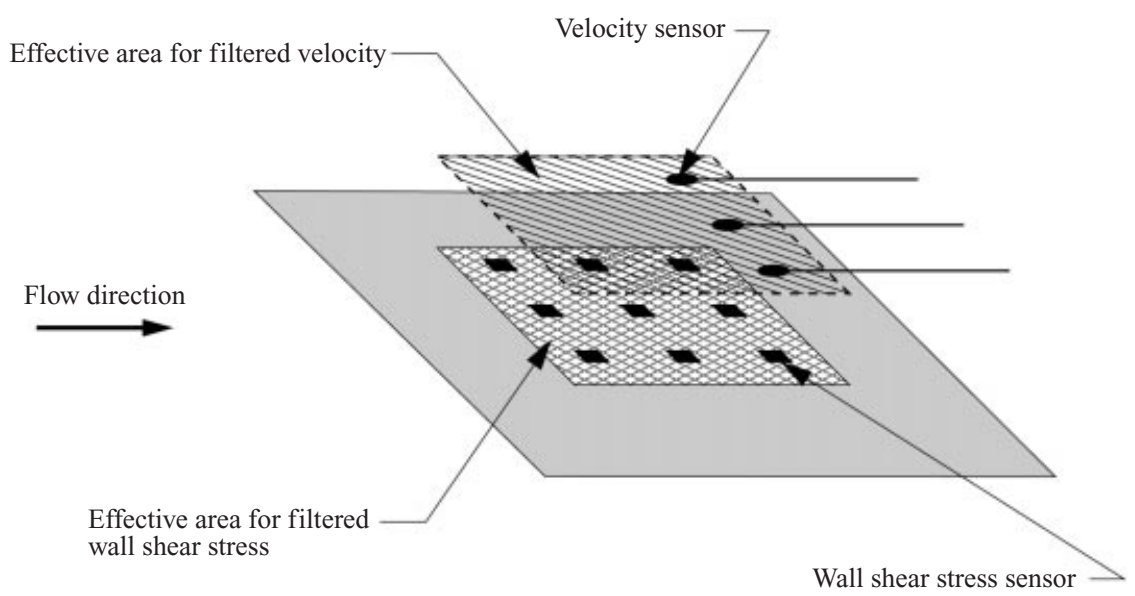

FIGURE 2. Schematic of experimental setup.

of each of the individual hot-film signatures was thus adjusted to the corresponding values obtained from the elevated hot wire. The correction was applied to each individual hot-film sensor before the two-dimensional filter was applied. A similar correction scheme was used by Ljus, Johansson \& Almstedt (2000). Here we are effectively neglecting any phase shifts associated with frequency response attenuation. However the correction seems to work particularly well. For example, the average relative root-mean-square with respect to the mean of the nine corrected shear stress signatures is 0.34 , while the average skewness and flatness are 0.9 and 4.7 respectively. Before correction these values were $0.06,0.7$ and 3.9 respectively. The corrected results agree well with the previous studies of Khoo et al. (1998) and Alfredsson et al. (1988). It is important to note that although all of the individual shear stress sensors have been corrected against one elevated hot wire the fluctuating characteristics of each individual hot film are still preserved.

\section{Results}

The experimental setup, shown schematically in figure 2, is designed to simulate a computational cell immediately above the wall in an LES. Consistent with high Reynolds number simulations, the filtered (resolved) velocity is obtained at a height where the logarithmic law of the wall holds. Similarly to Cerutti \& Meneveau (2000), Porté-Agel et al. (2001), and Tong, Wyngaard \& Brasseur (1999) a two-dimensional filter is used to approximate the three-dimensional filter applied in an actual LES. After applying Taylor's hypothesis, with a convection speed of $U_{c}=0.82 U_{1}$ for all positions considered (following Smits \& Dussauge 1996), a box filter of length $\Delta_{F}=18 \mathrm{~mm}$ (approximately 400 wall units) is applied in the streamwise and spanwise directions to obtain the filtered velocity. As in an actual LES the size of the filter is chosen to be larger than the vertical grid spacing. In the streamwise direction box, Gaussian, and sharp spectral filters, described in Pope (2000), were all examined but yielded negligible differences in the overall results. A two-dimensional filter of the same size $(18 \mathrm{~mm})$ is applied to the wall shear stress measurements. Note that a two-dimensional distribution of the wall shear stress sensors is required since Taylor's hypothesis cannot be used at the surface where the velocity is zero. The filtered wall shear stress obtained from the nine wall shear stress sensors will be referred to as 
the 'measured' wall shear stress and it will be compared to the 'modelled' wall shear stress obtained from the filtered velocity using equations (2.1), (2.2) and (2.3). These results are given below.

\section{1. $S G$ models}

A representative sample of the instantaneous modelled and measured wall shear stresses at $z^{+}=98$ is shown in figure $3(a)$. The correlation coefficient between the modelled, $\tau_{m}$, and measured, $\tau_{r}$, wall shear stress signature is defined as

$$
R_{\tau_{r} \tau_{m}}(T)=\frac{\left\langle\tau_{r}^{\prime}(t) \tau_{m}^{\prime}(t-T)\right\rangle}{\left(\left\langle\tau_{r}^{\prime 2}\right\rangle\left\langle\tau_{m}^{\prime 2}\right\rangle\right)^{1 / 2}},
$$

where primes signify a fluctuating quantity, and the results are shown in figure $3(b)$. Here $T$ is the time delay between the two signals which are both non-dimensionalized by their r.m.s. values. The $z^{+}$values shown correspond to positions in the boundary layer where the mean velocity profiles indicate that the logarithmic law of the wall applies. (Strictly, the position $z^{+}=311$ is slightly beyond the log region.) The corresponding power spectral densities of the modelled and measured wall shear stress fluctuations are shown in figure $3(c)$. Here

$$
\Phi_{\tau}(f)=\lim _{T \rightarrow \infty} \frac{1}{T}\left\{\mathrm{FT}^{*}\left[\tau^{\prime}(t)\right] \operatorname{FT}\left[\tau^{\prime}(t)\right]\right\},
$$

where $f$ is frequency, FT[ ] denotes the Fourier transform and * denotes a complex conjugate. All spectra are normalized such that

$$
\int_{0}^{\infty} \Phi_{\tau}(f) \mathrm{d} f=\left\langle\tau_{r}^{\prime^{2}}\right\rangle \quad \text { or } \quad\left\langle\tau_{m}^{\prime^{2}}\right\rangle .
$$

$\Phi_{\tau}\left(f / f_{c}\right)$ is defined as the power spectral density per non-dimensional frequency $f / f_{c}$, where $f_{c}=U_{c} /(2 \pi \delta)$ is taken to be a constant.

Note that the high-frequency oscillations, observed in the modelled shear stress spectra in figure $3(c)$, are a direct consequence of using a box filter but have negligible effects given the low energy content of the signals at these frequencies. Also the step observed in the measured shear stress spectrum indicates that some cross-correlation between the nine hot-film sensors exists at these frequencies. However, again at these frequencies the energy levels are negligible.

While figure 3(a) includes obvious discrepancies between the instantaneous signals, figures $3(b)$ and $3(c)$ quantify the statistical significance of the difference. The correlation results indicate only moderate levels of agreement with decreasing levels of peak correlation with increasing distance from the wall. Also, as the distance from the wall increases the peak in the correlation shifts to a greater delay. Overall these trends are consistent with the results of Brown \& Thomas (1977) and Rajagopalan \& Antonia (1979) who studied single-point shear stress and velocity correlations and is suggestive of coherent structures in the turbulent boundary layer. Using $U_{c}=0.82 U_{1}$ as the convection speed, the inclination angle of the inferred structures was found and agrees well with previous experiments of Brown \& Thomas (1977). It is because of this heightened correlation along structures that the shifted SG model was originally proposed.

The results in figure $3(c)$ indicate that the most significant discrepancy between modelled and measured shear stress spectra is in the lower energy content of the former indicating an underestimation of the r.m.s. value of the signal. The shapes of the modelled and measured spectra are reasonably similar, but it is noted that the 

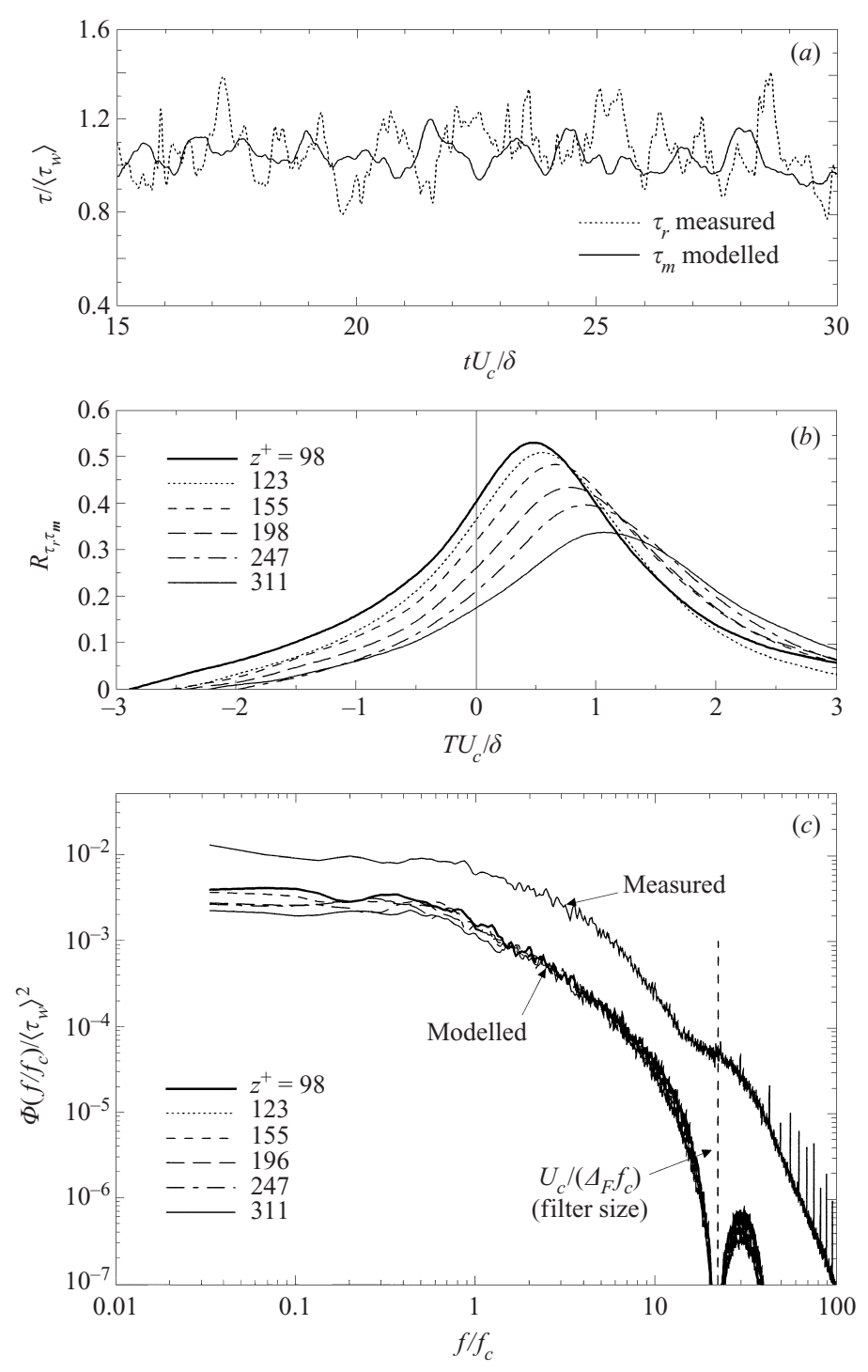

FIGURE 3. SG model wall shear stress comparison. (a) Typical sample of filtered wall shear stress signatures at $z^{+}=98$. (b) Correlation coefficient between modelled and measured wall shear stress. (c) Spectra of modelled and measured wall shear stress.

collapse of the individual modelled spectra is only moderately good and this may have implications for the SG model when applied to high Reynolds numbers. This will be further discussed in $\S 5$.

Shifting the velocity signature with the streamwise displacement $\Delta_{s}$ improves the correlation of the phases between modelled and measured shear stress signals as is expected and this is shown in figure 4 . The spectra will be unaffected by this shift.

\subsection{Ejection model}

The ejection model was proposed by Piomelli et al. (1989) in an attempt to better describe the shear stress at the wall associated with sweep-ejection events by using the wall-normal velocity. However, as shown in figure 5 the ejection model does a poorer 


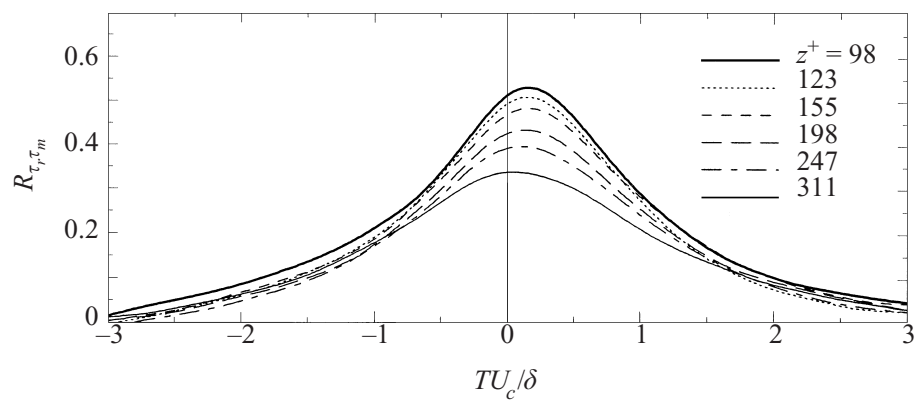

FIGURE 4. Shifted SG model wall shear stress comparison. Correlation coefficient between modelled and measured wall shear stress.

job of describing wall shear stress than the SG models. Here $C$, the dimensionless constant, was taken to be 0.6 which improves the agreement compared to using the suggested value of 1.0. The peak correlation coefficients are less than 0.25 and the spectra show significant differences in shape. As seen in figure $5(b)$, the modelled spectra generally have more weighting at higher frequencies. It is unclear whether the good agreement with the ejection model advocated by Piomelli et al. (1989), for their numerical study of channel flow at $R e_{\tau}=640$, indicates that low Reynolds number effects are significant. In the Piomelli et al. (1989) study no correlation coefficient nor spectral comparisons between measured and modelled wall shear stress were shown.

\section{Alternative model}

One of the difficulties in proposing a boundary condition model for LES is the restriction of only being able to use what is available during the simulation, that is, spatially filtered quantities. While it is unlikely that any model based purely on streamwise velocity at the first grid point can completely describe the streamwise component of the wall shear stress, the analysis of the previous three models suggests that using the streamwise component of the velocity is a better candidate than using the wall-normal component of velocity. However, these models still clearly have room for improvement. Two notable shortcomings have been revealed. First, the SG models do not adequately estimate the correct level of energy in the fluctuating components of the wall shear stress, and second, these models do not give equivalent results for the spectra at different wall-normal positions in the logarithmic region. This disagreement is expected to be worse for large changes in Reynolds numbers. Therefore in an attempt to overcome these two shortcomings, we propose a new model based on streamwise velocity

$$
\tau_{m}(x, y, z, t)=\left\langle\tau_{w}\right\rangle-\alpha U_{\tau}\left[\bar{u}\left(x+\Delta_{s}, y, z, t\right)-\langle\bar{u}(x, y, z, t)\rangle\right],
$$

where $\alpha$ is a characteristic constant.

The new model has been chosen such that the $\tau_{m}$-spectra will follow the filtered $u_{1}$-spectra and collapse to one curve for all positions in the logarithmic region for all Reynolds numbers. The underlying assumption here is that the low-frequency filtered $u_{1}$-spectra will follow regular $u_{1}$-spectra and collapse with 'outer-flow' scaling $\left(\Phi_{u_{1}}\left(f / f_{c}\right) /\left(U_{\tau}^{2}\right)=g\left(f / f_{c}\right)\right)$ as outlined in Perry, Henbest \& Chong (1986). Note that here using Taylor's hypothesis for the modelled stresses implies that $f / f_{c}=k_{1} \delta$, where $k_{1}$ is streamwise wavenumber. Furthermore, it is anticipated that the filtering operation will also collapse the data at the high frequencies. Figure 6 shows the results for the new model. (The correlation results will be identical to those of the shifted 

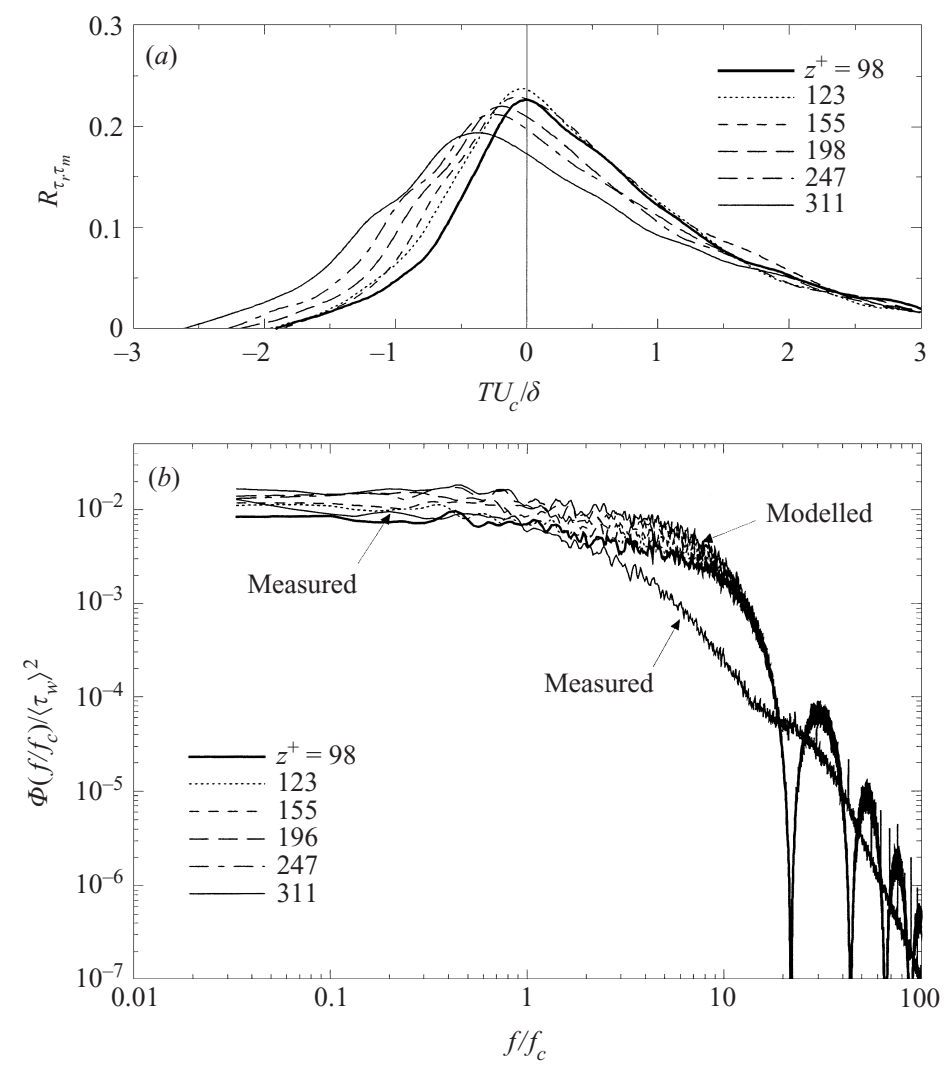

FIGURE 5. Ejection model wall shear stress comparison. (a) Correlation coefficient between modelled and measured wall shear stress. (b) Spectra of modelled and measured wall shear stress.

SG model.) The modelled spectra in figure $6(b)$ are seen to collapse very well. The small, but systematic, deviations at low non-dimensional frequencies are consistent with convection velocity variations not accounted for when using Taylor's hypothesis, as described by Perry \& Li (1991). Since the filtered $u_{1}$-spectra collapse, and hence follow the behaviour of regular $u_{1}$-spectra at low to moderate frequencies, it is likely that they will continue to do so for all Reynolds numbers.

The constant $\alpha$ was set at 0.10 for this zero-pressure-gradient flow. Support for 'outer-flow' scaling for $u_{1}$-spectra in the logarithmic region in a range of pressure gradient flows also exists. See for example, Marusic (1991) and Marusic \& Perry (1995) for adverse pressure gradient flows and Jones, Marusic \& Perry (2001) for favourable pressure gradient data. The level at which the spectra collapse is dependent on pressure gradient, and therefore this is why $\alpha$ has been termed a characteristic constant. Future work will determine what functional form the constant will take but current knowledge suggests its dependence on geometry and pressure gradient will be relatively weak.

Note that while figure 6(b) shows the desired collapse of the individual modelled $\tau_{m}$-spectra, the agreement with the measured wall shear stress spectra is not ideal across all the energy-containing frequencies. This is a limitation of using any model based on streamwise velocity alone. 

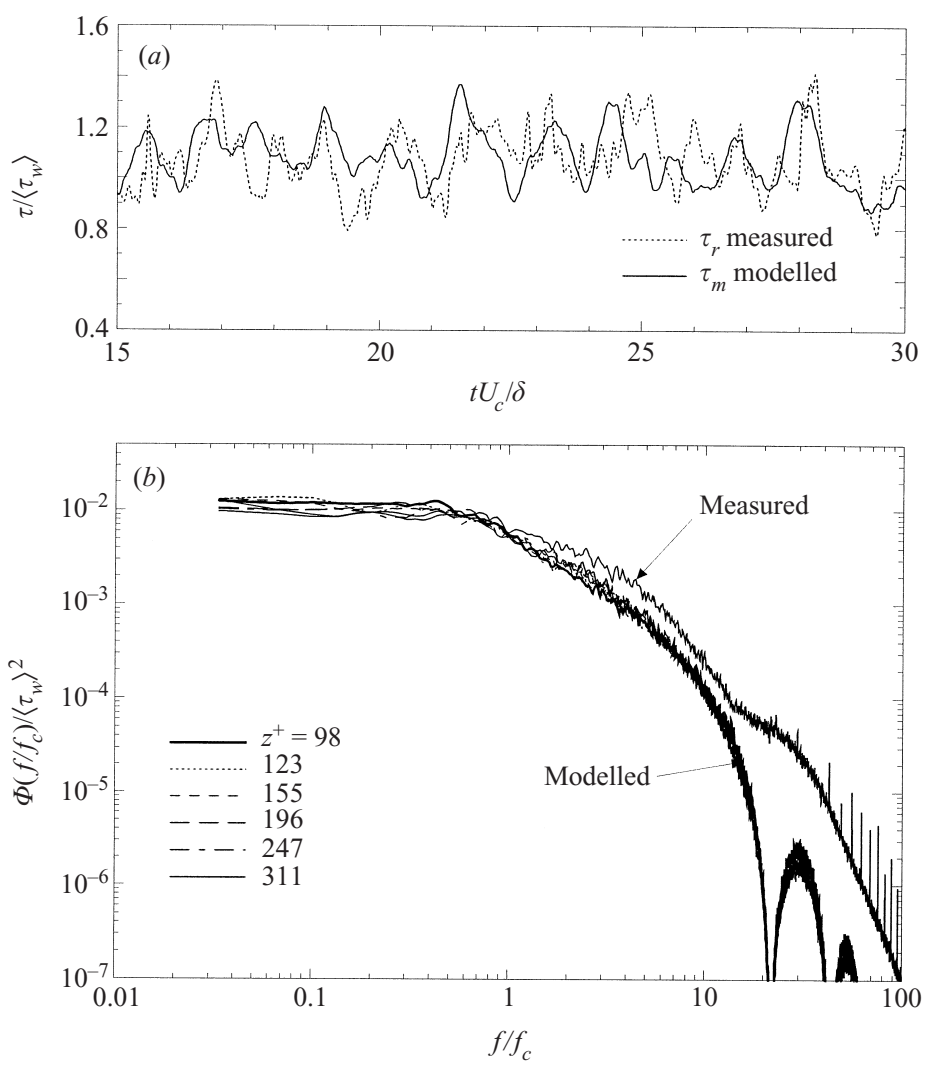

FIGURE 6. New model wall shear stress comparison. (a) Typical sample of filtered wall shear stress signatures at $z^{+}=98$. (b) Spectra of modelled and measured wall shear stress.

\section{Conclusions}

In this experimental study, three existing models were tested for the estimation of wall boundary conditions for LES in a turbulent boundary layer. The study was confined to the streamwise component of the instantaneous wall shear stress and the first grid point was taken to be within the region of the flow where the logarithmic law of the wall holds. This is consistent with a high Reynolds number simulation. Two of the models (SG and shifted SG) are based on the streamwise velocity and were found to give better results than the ejection model, which uses the wall-normal component of velocity. Peak normalized correlations between measured and modelled filtered wall shear stress were in the range $0.53-0.34$ for the SG models, with the correlation decreasing with increasing distance from the wall. The corresponding correlation coefficients for the ejection model were in the range 0.24-0.19.

A new model is proposed which is also based on the streamwise velocity at the first grid point. The model gives better agreement with the measured wall shear stress than either of the SG models. It is believed that this is the best model that can be proposed based on one-point information of the filtered streamwise velocity. The new model incorporates the attractive features of the shifted SG model, thereby accounting for inclined coherent structures near the wall. However, the main feature of the new model is that it has been chosen such that the modelled spectra will agree with each other for any positions within the logarithmic region at any Reynolds number. This comes from the fact that the corresponding streamwise 
velocity spectra follow 'outer-flow' scaling similarity as described by Perry et al. (1986). The proposed model is simple enough to be used directly in existing LES codes, and it is intended for attached boundary layers where the mean wall shear stresses are known.

The authors gratefully acknowledge the financial support of the National Science Foundation, NASA and the University of Minnesota Grant-in-Aid program. IM was supported under NSF grants CTS-9983933 and ACI-9982274 and FPA was supported under NSF grant EAR-0094200 and NASA grant NAG5-10569.

\section{REFERENCES}

Adrian, R., Meneveau, C., Moser, R. \& Riley, J. 2000 Final report on 'Turbulence Measurements for LES' workshop. Tech. Rep. 937. Theoretical and Applied Mathematics, University of Illinois at Urbana-Champaign.

Alfredsson, P. H., Johansson, A. V., Haritonidis, J. H. \& Eckelmann, H. 1988 The fluctuating wall-shear stress and the velocity field in the viscous sublayer. Phys. Fluids 31, 1026-1033.

BREUER, K. S. 1995 Stochastic calibration of sensors in turbulent flow fields. Exps. Fluids 19, $138-141$.

Brown, G. L. \& Thomas, A. S. W. 1977 Large structure in a turbulent boundary layer. Phys. Fluids 20, S243-S252.

CАвот, W. \& Moin, P. 2000 Approximate wall boundary conditions in the large-eddy simulation of high Reynolds number flow. Flow, Turbulence Combust. 63, 269-291.

Cerutti, S. \& Meneveau, C. 2000 Statistics of filtered velocity in grid and wake turbulence. Phys. Fluids 12, 1143-1165.

Chew, Y. T., Khoo, B. C., Lim, C. P. \& Teo, C. J. 1998 Dynamic response of a hot-wire anemometer. Part II: A flush-mounted hot-wire and hot-film probes for wall shear stress measurments. Meas. Sci. Technol. 9, 764-778.

Grötzвасн, G. 1987 Direct numerical and large eddy simulations of turbulent channel flows. Encyclopedia of Fluid Mechanics (ed. N. P. Cheremisinoff), vol. 6, pp. 1337-1391. Gulf.

Jones, M. B., Marusic, I. \& Perry, A.-E. 2001 Evolution and structure of sink-flow turbulent boundary layers. J. Fluid Mech. 428, 1-27.

Khoo, B. C., Chew, Y. T., Lim, C. P. \& Teo, C. J. 1998 Dynamic response of a hot-wire anemometer. Part I: A marginally elevated hot-wire probe for near-wall velocity measurments. Meas. Sci. Technol. 9, 751-763.

Luus, C., Johansson, B. \& Almstedt, A.-E. 2000 Frequency response correction of hot-film measurements in a turbulent airflow. Exps. Fluids 29, 36-41.

MARUSIC, I. 1991 The structure of zero- and adverse-pressure gradient turbulent boundary layers. $\mathrm{PhD}$ thesis, University of Melbourne, Australia.

Marusic, I. \& Perry, A. E. 1995 A wall-wake model for the turbulence structure of boundary layers. Part 2. Further experimental support. J. Fluid Mech. 298, 389-407.

Perry, A. E., Henbest, S. M. \& Chong, M. S. 1986 A theoretical and experimental study of wall turbulence. J. Fluid Mech. 165, 163-199.

Perry, A. E. \& Li, J. D. 1991 Theoretical and Experimental studies of shear stress profiles in two dimensional turbulent boundary layers. Rep. FM-18. Dept. of Mech. Eng., University of Melbourne.

Piomelli, U., Ferziger, J., Moin, P. \& Kim, J. 1989 New approximate boundary conditions for large eddy simulations of wall bounded flows. Phys. Fluids A 1, 1061-1068.

Pope, S. B. 2000 Turbulent Flows. Cambridge University Press.

Porté-Agel, F., Parlange, M. B., Meneveau, C. \& Eichinger, W. E. 2001 A priori field study of the subgrid-scale heat fluxes and dissipation in the atmospheric surface layer. J. Atmos. Sci. In press.

Rajagopalan, S. \& Antonia, R. A. 1979 Some properties of the large structure in a fully developed turbulent duct flow. Phys. Fluids 22, 614-622. 
Schumann, U. 1975 Subgrid scale model for finite difference simulations of turbulent flows in plane channels and annuli. J. Comput. Phys. 18, 376-404.

Smits, A. J. \& Dussauge, J. P. 1996 Turbulent Shear Layers in Supersonic Flow. AIP Press.

Tong, C. N., Wyngaard, J. C. \& Brasseur, J. G. 1999 Experimental study of the subgrid-scale stresses in the atmospheric surface layer. J. Atmos. Sci. 56, 2277-2292. 


\section{University Library}

\section{- M M I E R R A A gateway to Melbourne's research publications}

Minerva Access is the Institutional Repository of The University of Melbourne

Author/s:

Marusic, I.;Kunkel, G. J.;Porte-Agel, F.

Title:

Experimental study of wall boundary conditions for large-eddy simulation

Date:

2001

Citation:

Marusic, I., Kunkel, G. J. \& Porte-Agel, F. (2001). Experimental study of wall boundary conditions for large-eddy simulation. Journal of Fluid Mechanics, 446, 309-320.

Publication Status:

Published

Persistent Link:

http://hdl.handle.net/11343/34791 